\title{
ASTEROID SURFACE MATERIALS FROM REFLECTANCE SPECTROSCOPY: A REVIEW
}

\author{
M. J. GAFFEY and T. B. McCORD
}

The quality and quantity of asteroidal spectral reflectance data has increased rapidly in recent years. This data can be either (a) classified into spectral, polarization and/or albedo groups (e.g., ' $C$ ', 's', etc.) which should tend to lump together grossly similar materials, or (b) interpreted utilizing laboratory and theoretical understanding of spectral features (e.g., absorption bands, continuum shape and slope) which are diagnostic of mineralogy or mineral chemistry. Mineral assemblages have been identified on asteroid surfaces which are comparable to most known meteorite types or which have undergone the types of processes (e.g., melting and differentiation) necessary to produce the meteoritic assemblages. The ordinary chondrites, which dominate the meteoritic flux reaching the Earth's surface, are very rare or absent on Main Belt asteroids but appear common on the small asteroids which approach or cross the orbit of the Earth. The present interpretations of asteroidal spectra are not yet quantitative enough to permit the evaluation of specific asteroids as the sources bodies of particular meteorite specimens in terrestrial collections.

\section{INTRODUCTION}

The last decade has witnessed a dramatic increase in the number of asteroids for which spectral data of a given resolution or wavelength coverage is available. In a review of the spectrophotometric studies of asteroids up to 1970 (Chapman, Johnson and McCord 1971), it was noted that complete UBV colors for 36 asteroids were available in the literature (e.g., Gehrels 1970). In the relatively narrow bandpass 24 filter system $(\sim 0.3-1.1 \mu \mathrm{m})$ of McCord, Chapian and their co-workers, spectral data was available for 12 asteroids. At the end of 1975, UBV colors were available for 91 asteroids (Zellner, Wisniewski, Andersson and Bowell 1975) and narrow-band spectrophotometric measurements were avai1able for nearly 100 asteroids (Chapman, McCord and Johnson 1973, Chapman, McCord and Pieters 1973, McCord and Chapman 1975a,b, Pieters, Gaffey, Chapman and McCord 1976). Furthermore, observational work has been initiated for the $1-3 \mu \mathrm{m}$ region with the broad-band JHK filters $(1.25,1.65$ and $2.2 \mu \mathrm{m}$ respectively) for more than 25 asteroids (Johnson, Matson, Veeder and Loer 1975, Matson, Johnson and Veeder 1976, Chapman and Morrison 1976) and higher resolution interferometric observations of three bright asteroids: 4 Vesta-Larson and Fink (1975), 433 Eros-Larson, Fink, Treffers and Gautier (1976) and 1 Ceres-Larson (1976). These data sets are shown schematically with respect to spectral coverage and resolution at the top of Figure 1 . 


\section{GAFFEY AND MCCORD}

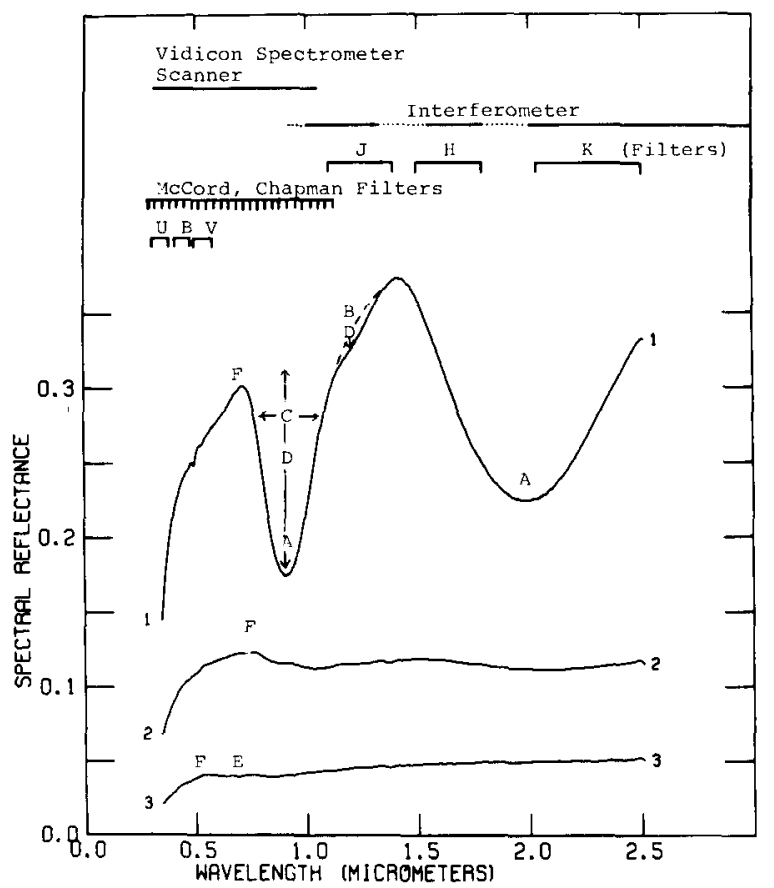

Figure 1. The wavelength coverage (solid lines) and spectral resolution eiements (bounded by tic marks) of the available asteroid spectral data sets is compared to the spectral reflectance curves of three moteorite classes which are similar to certain asteroid surface materials: (1) eucrite (pyroxene-plagioclase achondrite), (2) C30 (CO) carbonaceous chondrite (olivine + carbon + magnetite) and (3) C2 (CM) carbonaceous chondrite (clay minerals + carbon). The important spectral parameters which must be established for precise interpretation of such spectra are: absorption band positions of pyroxene (A) and plaginclase feldspar (B) which provide mineral chemistry data, symmetry of 1 um band (C) which provides measure of olivine/pyroxene ratio, band intensities of pyroxene and plagioclase features (D) which provides a measure of pyroxene/plagioclase ratio, position and intensity of $0.6 \mathrm{~nm} \mathrm{Fe} \mathrm{Fe}^{2+}$ $-\mathrm{Fe}^{3+}$ or $\mathrm{Fe}^{3+}$ features (E) for clay mineralogy and oxidation state. overaIl spectral contrast $(\sim \Delta R e f / \Delta \lambda)$ which provides measure of relative abundance of opaque and non-opaque'phases and wavelength position of break in slope from visible-IR to bIue-UV falloff ( $F$ ) which wher considered with albedo data provides a measure of the spectral optical density of non-opaque phase.

The ability to extract information concerning the composition of asteroid surface materials from this spectral data has aiso evolved over this interval. Early efforts to match observational data with laboratory spectral measurements of possible comparison materials, especially meteorites, are giving way to interpretations based on the identification of spectral features which are diagnostic of the presence and composition of specific mineral phases. These two approaches provide different information about the surface materials responsible for a particular spectral curve.

\section{DESCRIPTIONS OF ASTEROID SURFACE MATERIALS - SPECTRAL MATCHING}

This approach characterizes asteroid surface materials by a direct compar- 
ison of the observational data to appropriate laboratory measurements of possible comparison materials. For example, Hapke (1971) compared the UBV colors of a number of asteroids to a variety of lunar, meteoritic and terrestrial rocks and rock powders. The conclusion of this comparison was that the surface material of these asteroids could be matched by powders similar to a range of the comparison materials but not by metallic surfaces. It should be noted that with this type of comparison, one is selecting for materials which are similar with respect to the chosen parameters (e.g., UBV colors), which may or may not be mineralogically significant.

The sophistication of any matching approach increases as the number of parameters included in the comparison increases. Thus, Chapman and Salisbury (1973) and Johnson and Fanale (1973) by comparing narrow-filter, 0.3-1.1 um asteroidal spectra to laboratory meteorite spectra were able to make much more specific statements concerning the asteroid surface materials. Chapman and Salisbury (1973) and Johnson and Fanale (1973) both noted the problem of defining precisely what constituted a 'match,' and both raised the question of subtle spectral modification of asteroid surface materials by in situ space weathering processes. Salisbury and Hunt (1974) raised the question of the effects of terrestrial weatharing on meteorite specimens and the validity of matches between the spectra of such specimens and the asteroids. Hapke (1971) had noted the significant difference in the UBV colors between a rock and powders of that rock. With respect to the UBV colors the data presented by Hapke (1971) and Zellner et al. (1975) show a significant degree of redundancy for distinctly different meteorite types. That is, the regions defined on a UBV color plot by several quite discrete meteorite types may overlap or overlay each other. Since the matching approach does not depend on any significant understanding of the mineralogical basis for the spectral behaviour of a material, the lack of such understanding does not limit the utilization of this approach. However, the lack of such understanding does increase the difficulty in evaluating the significance of the deviation from a precise match and in evaluating the uniqueness of such a match as a description of an asteroid surface material.

This difficulty can be somewhat alleviated by simultaneously matching nonspectral (e.g., albedo and polarization) and spectral parameters for the asteroids and comparison materials. Chapman, Morrison and Zellner (1975) utilized this approach to define two broad groups which include most asteroids. The first group was characterized as having low albedos ( 20.09$)$, strong negative polarizations at small phase angles $(>1.1 \%)$ and relatively flat, featureless spectral reflectance curves. These parameters were similar to those for carbonaceous chondrites and these asteroids were designated as 'carbonaceous' or ' $C$ ' type. The second group was characterized as having higher albedos $(50.09)$, weaker negative polarizations $(0.4-1.0 \%)$ and reddish, featured spectra1 curves. These parameters were comparable to those for most of the meteorites which contain relatively abundant silicate minerals, so this group was designated 'silicaceous or stony-iron' or ' $S$ ' types. A small minority $(-10 \%)$ of the asteroids could not be classified in this system and were designated 'unclassified' or ' $U$ ' types.

In at least one way this was an unfortunate choice of terminology, since it implies a specific definition of surface materials which was not intended. Thus any 'flat-black' spectral curve would be designated ' $C$ ' type whether or not the surface material would be characterized as carbonaceous by any other criteria. Thus the meteoritic materials such as ureilites, black chondrites and the non'carbonaceous' C4 meteorite 'Karoonda' would all be lumped under the label 'carbonaceous,' with the corresponding invalid implication concerning their composition and/or parentage.

The problem is complicated by the terminology for the meteorites themselves. As Mason (1971) noted, the term 'carbonaceous' immediately implies the 
presence of carbon or carbonaceous compounds as a distinctive component. However, a number of the carbonaceous chondrites, especially types C3 and C4, contain less carbon than many non-'carbonaceous' meteorites. (Indeed, the presently accepted definition of the carbonaceous chondrites has little or nothing to do with carbon $(e . g ., \mathrm{Mg} / \mathrm{Si}>1.0$ (atomic) - Van Schmus and wood 1967)). It is essential to realize that the term 'carbonaceous asteroid' is not necessarily equivalent to 'carbonaceous meteorite' (as broad as that latter classification is), that 'carbonaceous' is a spectral designation with respect to asteroids and a compositional designation with respect to meteorites. The same objection can be raised with respect to the 'silicaceous' terminology since it implies a degree of specificity not present. It must also be noted the criticism here is with respect to terminology and the confusion it engenders and not with respect to the identification of these two major groups, which appear to be significant with respect to the parameters chosen.

The value of a matching approach in characterizing asteroid surface materials is that it can be applied to data with minimal spectral coverage and/or resolution to provide valid information within its limitations. It is thus very valuable as a survey technique to rapidly investigate a large number of objects and to select targets for more sophisticated techniques (e.g., Zellner 1975). The UBV data is especially important because the broad bandpass filters in the visible portion of the spectrum provide a high photon flux at any magnitude relative to other narrower bandpass systems. This permits UBV measurements to be made to much fainter magnitudes, thus providing some information concerning objects which otherwise would go unsampled. Care must be taken to avoid abusing such data by over-interpretation.

\title{
DESCRIPTIONS OF ASTEROID SURFACE MATERIALS - INTERPRETATION OF DIAGNOSTIC
}

\author{
SPECTRAL FEATURES
}

The alternate approach to characterizing asteroid surface materials involves the interpretation of features in a reflectance spectrum which are diagnostic of specific minerals. This interpretive approach can provide a much more definitive description of a surface material since it utilizes welldefined criteria to establish the presence or absence of a specific mineral phase. This approach provides (within limits) definite mineralogical and petrological descriptions of surface materials while the matching approach suggests materials which may be similar to an asteroid surface. But while the matching technique can be applied to virtually any spectral data to provide at least some information, the interpretative technique requires spectral data with a certain minimum spectral coverage and resolution so that the presence (or absence) and parameters of the spectral features can be established. There is the additional requirement for at least a qualitative understanding of the physics involved in producing a particular spectral feature and thus its significance. A large amount of background laboratory and theoretical work is necessary to reach such an understanding.

\section{THEORETICAL BASIS FOR INTERPRETATION OF DIAGNOSTIC SPECTRAL FEATURES}

Since the early 1960's a major effort has been underway to define the physics which govern the interaction of light with the common rock-forming silicate minerals (e.g., White and Keester 1966, 1967, Burns 1970b, Be11, Mao and Rossman 1974). This work (crystal field theory, ligand field theory or molecular orbital theory) has been summarized for mineralogical systems by Burns (1970a).

Elements of the first transition series ( $\mathrm{Sc}, \mathrm{Ti}, \mathrm{V}, \mathrm{Cr}, \mathrm{Mn}, \mathrm{Fe}, \mathrm{Co}, \mathrm{Ni}, \mathrm{Cu}$ ) and their petrologically important cations (esp. $\mathrm{Fe}^{2+}, \mathrm{Ti}^{3+}$ ) have an outer unfilled $d$-shell in their electron distribution. The electron distributions for 


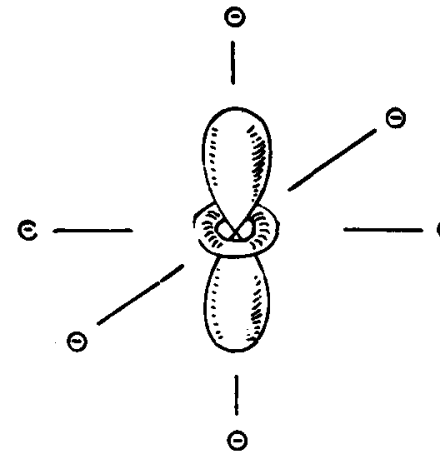

$d_{z^{2}}$

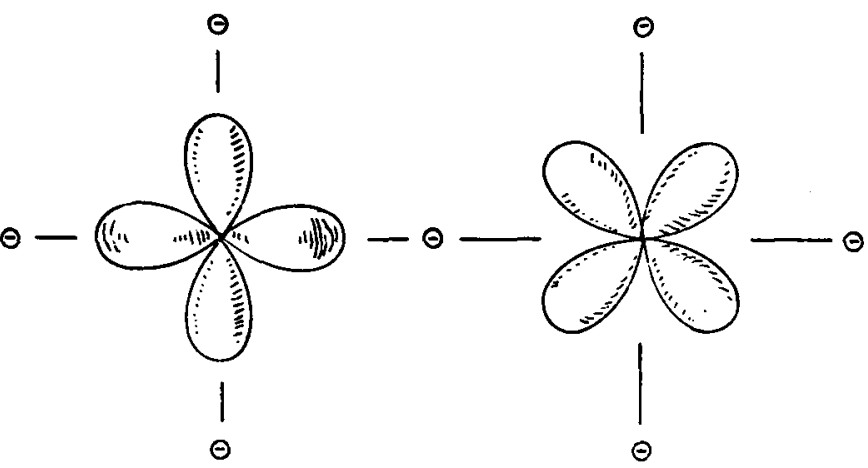

$x^{2}-y^{2}$
$d_{X Y}, d_{X Z}, d_{Y Z}$

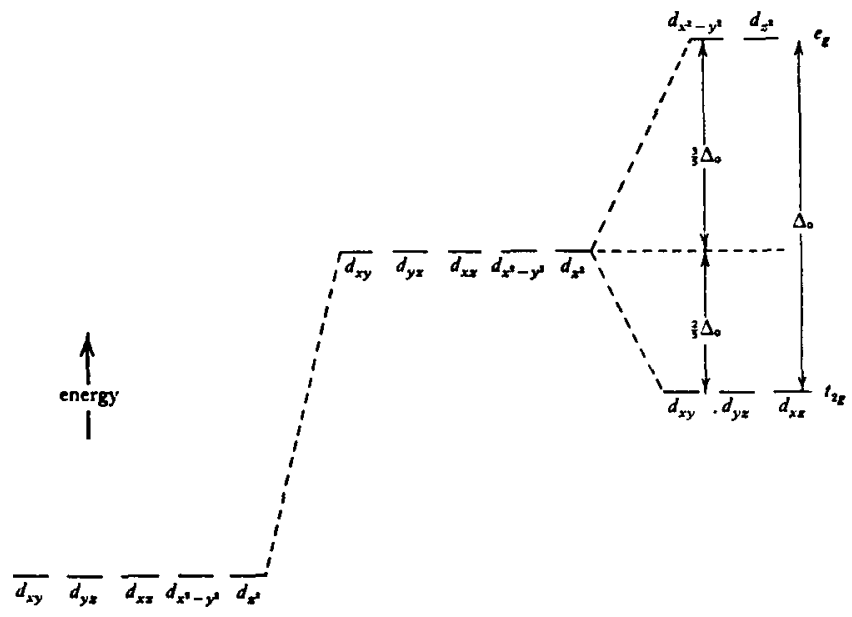

free ion

unperturbed ion in a crystal field ion in an octahedral crystal field

Figure 2. (a) Distribution of electron probability for d-orbitals of transition metal cation (e.g., $\mathrm{Fe}^{2+}$ ) in octahedral crystal lattice site defined by location and symmetry of six surrounding anions. (b) Relative energy levels of the d-orbitals of a transition metal cation as a free ion, as an unperturbed ion in a crystal field and the crystal field splitting in a octahedral site (reproduced from Burns (1970a)).

the five possible $d$-shells are shown on Figure $2 \mathrm{a}$. When such a cation is located in a crystal site surrounded by anions (shown schematically on Figure 2a), certain orbitals experience strong electronic repulsions and undergo splitting to higher energy. The orbitals undergoing the least electronic repulsion become the groundstate orbitals in which the electrons tend to reside (Figure 2b). The energy difference between the groundstate and any excited state is termed the crystal field splitting energy and is a direct function of particular cation and the crystal site in which it resides. A photon whose energy corresponds to the 
splitting energy of a particular cation can be absorbed. This gives rise to specific absorption features in the reflectance spectra of transition-metal sili-

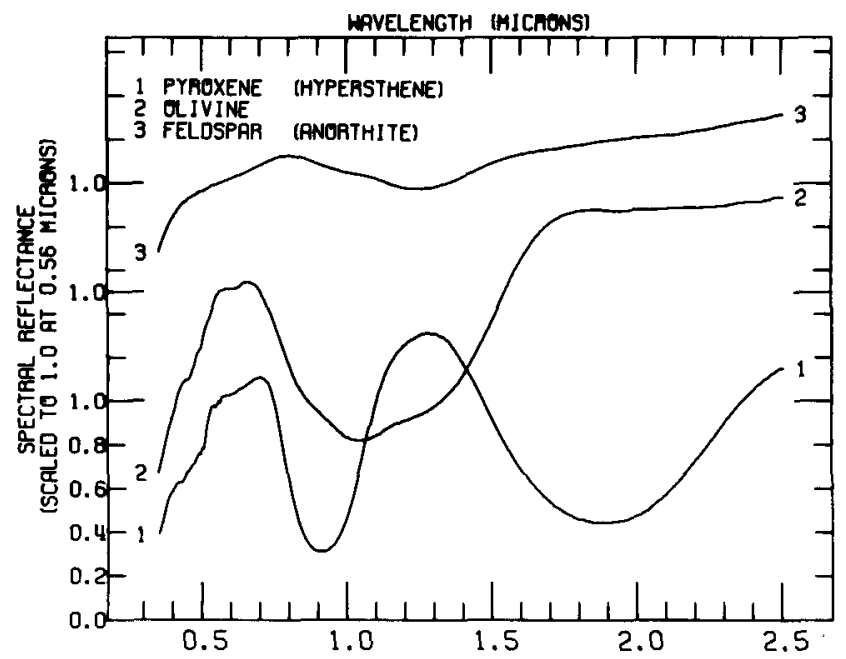

Figure 3. Nomalized spectral reflectance curves for pyroxene (bands near 1 and $2 \mu \mathrm{m}$ ), olivine (broad, asymmetric, three-lobed band near $1 \mu \mathrm{m}$ ) and feldspar $\left(\sim 1.25 \mu \mathrm{m} \mathrm{Fe}^{2+}\right.$ band).

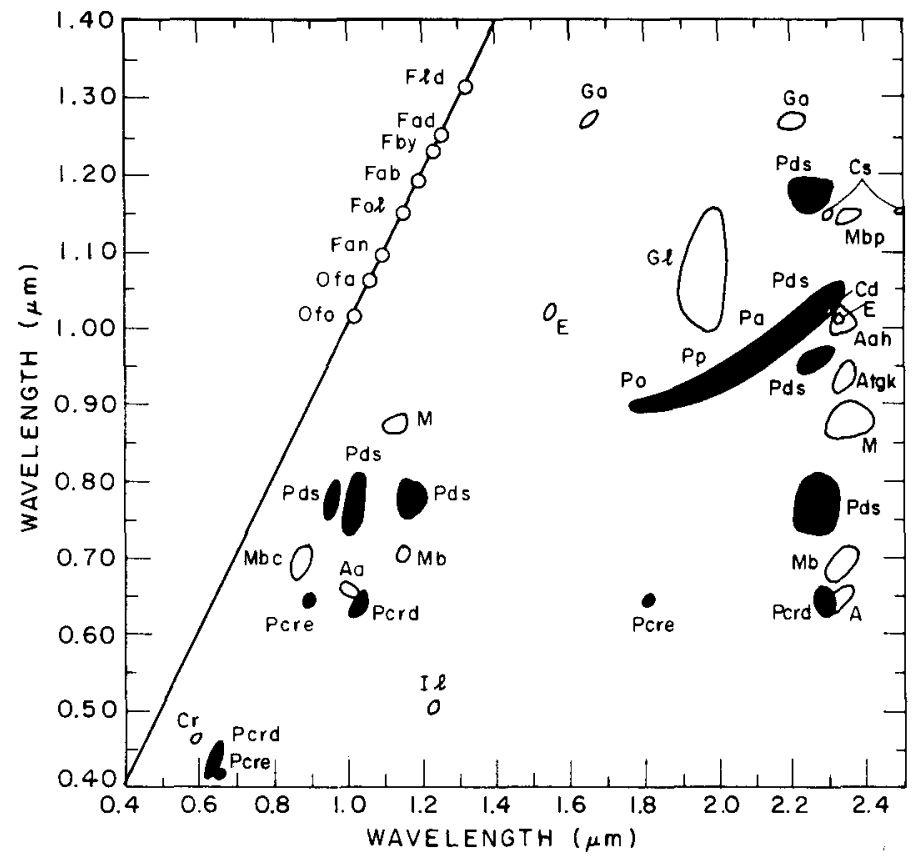

Eigure 4. Band-Band plot for minerals and glasses (Adams 1974). Note the dispersion of different mineral types and compositions. Spectia with a single band are shown along bottom of figure. 
ASTEROID SURFACES FROM SPECTRA
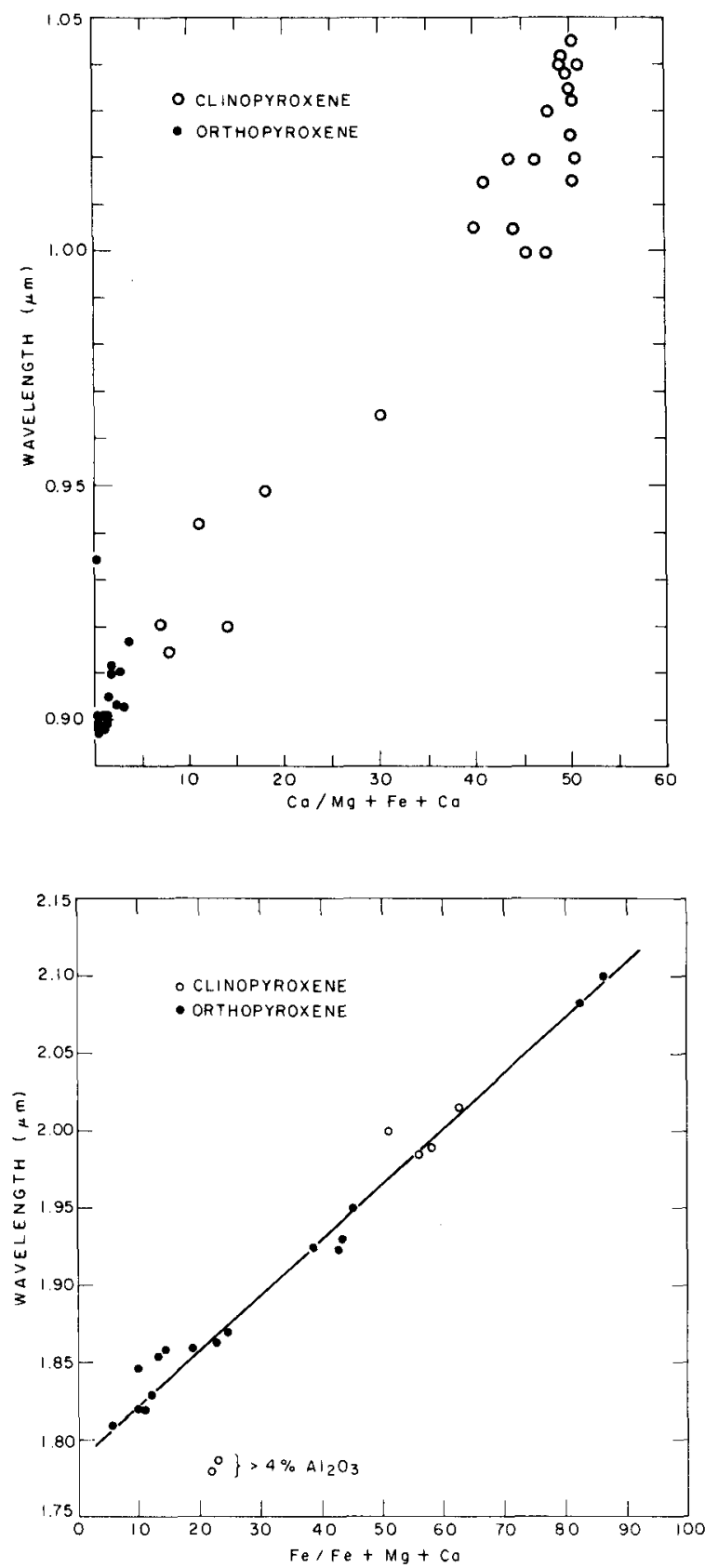

Figure 5. (a) Calcium content of ortho- and clinopyroxenes as a function of Band I position. (b) Iron $\left(\mathrm{Fe}^{2+}\right)$ content of ortho- and clinopyroxenes as a function of Band II position. (Adams 1974). 


\section{GAFFEY AND MCCORD}

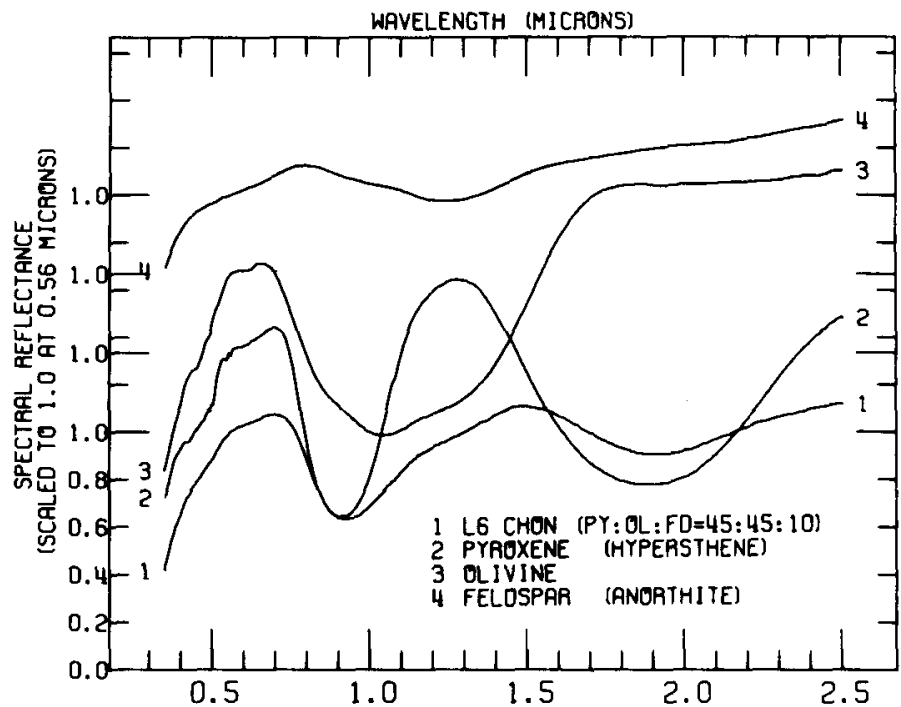

Figure 6. Normalized reflectance spectra of mixture (L6 chondrite) and individual silicate phases. Note that the mixture spectral curve is basically a pyroxene curve with the long wavelength wing of Band I depressed by the olivine and a waak 'nick' in the curve near $1.3 \mu \mathrm{m}$ from the feldspar feature (Gaffey 1976).

cates such as olivine, pyroxene and feldspar (Figure 3). Adams (1974) has shown that the positions of these bands is distinctive for each type of mineral (Figure 4). Adams (1974) has calibrated the precise positions of the two pyroxene bands with respect to the mineral chemistry of the specimens (Figure $5 \mathrm{a}$ and $5 \mathrm{~b}$ ). Adams (1976, personal communication) is working to establish a similar calibration for the $1.25 \mu \mathrm{m} \mathrm{Fe}{ }^{2+}$ absorption feature in feldspar.

Quantification of the spectral parameters of mineral fixtures in order to be able to interpret reflectance spectra for mineral abundance as well as composition, is a more complex problem. For example, Adams and McCord (1970), Nash and Conel (1974) and Gaffey $(1974,1976)$ have noted that the relative abundance of a mineral phase does not correlate directly with its apparent spectral abundance. Johnson and Fanale (1973) and Nash and Cone1 (1974) used laboratory mixtures to show that an opaque phase (carbon and magnetite, respectively) would dominate the reflectance spectrum of a mixture even when present in small amounts. Gaffey (1976) (Figure 6) showed how the relatively more optically dense pyroxene phase dominates the spectrum of a pyroxene-olivine-feldspar assemblage (L6 chondrite), but that the pyroxene spectrum is modified by the olivine and feldspar features. The olivine feature tends to lower the reflectance of a mixture near $1 \mathrm{\mu m}$, distorting the long wavelength wing of the $0.9 \mathrm{\mu m}$ pyroxene feature. This distortion of the combination olivine-pyroxene feature near $1 \mu \mathrm{m}$ can be calibrated to the relative abundance of these two phases as shown on Figure 7 (Gaffey 1976, in preparation). Computer deconvolution of spectra can be used to suppress the continuum and strong features in order to enhance weak absorption features such as that of feldspar (Figure 8) and the relative intensity of the pyroxene and feldspar absorption features can be correlated to the relative abundance of these mineral phases (Figure 9) (Gaffey 1976, in preparation).

The preceding diagnostic spectral features can be termed specific since they are related to specific minerals. For a number of minerals such specific diag- 


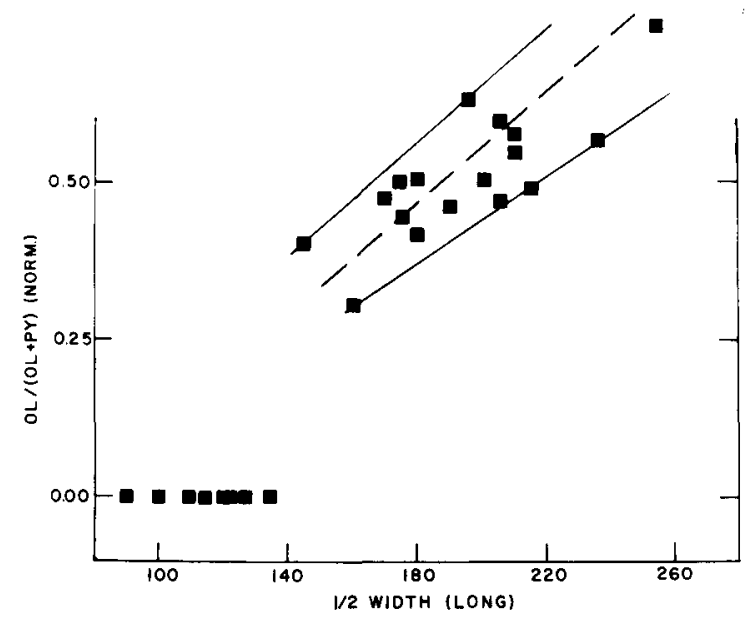

Figure 7. Correlation of half width at half height the long-wavelength wing of Band I for olivine + pyroxene mixtures versus olivine/pyroxene ratio. (Gaffey 1976, in preparation).

nostic features are absent or very weak. The general spectral behavior can provide a degree of diagnostic capability for such materials.

The slope and linearity or curvature of the visible and near-IR spectral continuum is such a general spectral parameter. The spectral reflectance curve of a $\mathrm{Ni}-\mathrm{Fe}$ metal in energy space $\left(i . e, \mathrm{Cm}^{-1}\right)$ in the visible and IR $(14,000-$ $30,000 \mathrm{~cm}^{-1}$ or $0.3-0.7 \mu \mathrm{m}$ ) is quite linear (Figure 10a). The reflectance is inversely proportional to the frequency of the incident photon.

For a transition metal silicate, the probability of a photon being absorbed increases rapidly in the blue and $U V$ portions of the spectrum due to increasingly efficient interionic charge transfer absorptions at higher energies. This produces a strongly curved continuum with a strong UV falloff (Figure 10b). Even in the case of the $\mathrm{C} 3$ chondrites which are spectrally dominated by the presence of opaque phases (Johnson and Fanale 1973, Gaffey 1976), the curved continuum characteristic of the silicates is evident (Figure 10c).

For an assemblage with multivalent transition metal ions (e.g., $\mathrm{Fe}^{2+}$ and $\mathrm{Fe}^{3+}$ ) extremely strong charge transfer absorptions dominate in the blue portion of the spectrum. The sharp decrease in the reflectance of the $\mathrm{Cl}$ and $\mathrm{C} 2$ carbonaceous chondritic assemblages (opaques plus $\mathrm{Fe}^{2+}-\mathrm{Fe}^{3+}$ clay mineral silicates) is the result of the very large increase in optical density due to this intense charge transfer absorption, overwhelming even the effect of the opaque phase (Figure 10d).

Such general spectral behavior provides an indication of the general minerologic group to which a material must belong, but cannot specify the particular mineral involved. Continuous effort in laboratory and theoretical investigations of such materials promises to improve this situation.

As a general rule, the sophistication of the interpretation of a reflectance spectrum is a function of the spectral resolution, spectral coverage and signalto-noise of the observationa $i$ data. This is graphically illustrated by comparing the top and bottom portions of Figure 1. The location of the mineralogically significant spectral features (as discussed above) can be compared to the location and bandpass of the several data sets. It is evident that relatively high resolution $(-100-300 \AA)$ for the $0.4-1.0 \mu \mathrm{m}$ region is the minimum data set which can provide any significant mineralogical characterization of an asteroid 


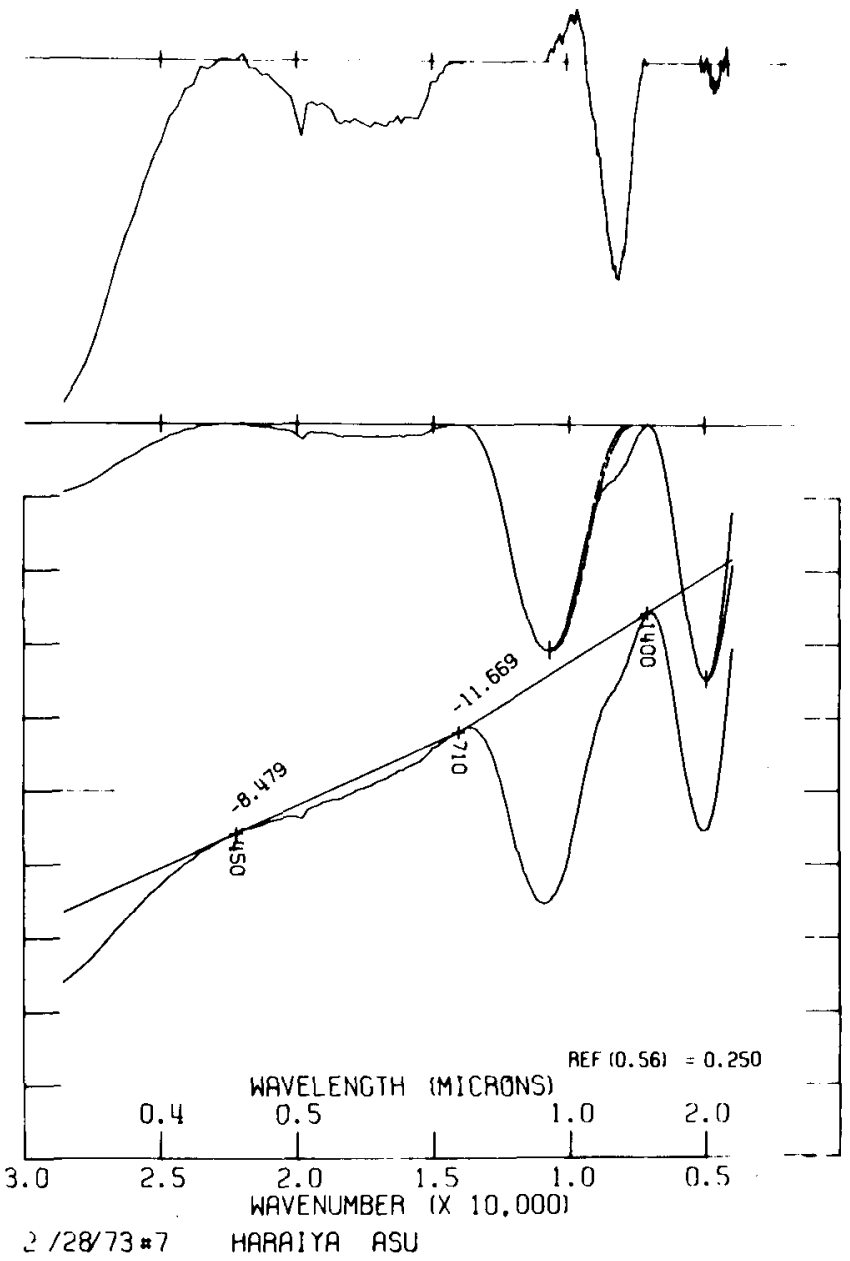

Figure 8. Computer deconvolution of spectral curve of pyroxene-plagioclase mixture, involving establishment of empirical continuum (A) for criginal curve $(B)$, continuum minus spectrum (C), spectral features are mirrored about center of feature and subtracted to isolate weak feldspar band (D). (Gaffey 1976, in preparation).

surface material. Increased resolution and spectral coverage significantly improve the interpretation. (For example, the narrow, weak spin-forbidden absorption feature at $0.5 \mu \mathrm{m}$ characteristic of specific pyroxenes, and which should be present in the spectrum of the asteroid 4 Vesta, cannot be resolved, let alone characterized by any presently available observational data set).

The evolving interpretation of the surface mineralogy of the asteroid 4 vesta is illustrative of the improving sophistication of the interpretative process.

a) McCord, Adams and Johnson (1970) measured the reflectance spectrum of Vesta with moderate spectral resolution and coverage $(0.40-1.08 \mu \mathrm{m}, \sim 24$ filters). They identified a deep absorption band $(\sim 0.92 \mu \mathrm{m})$ which they inter- 


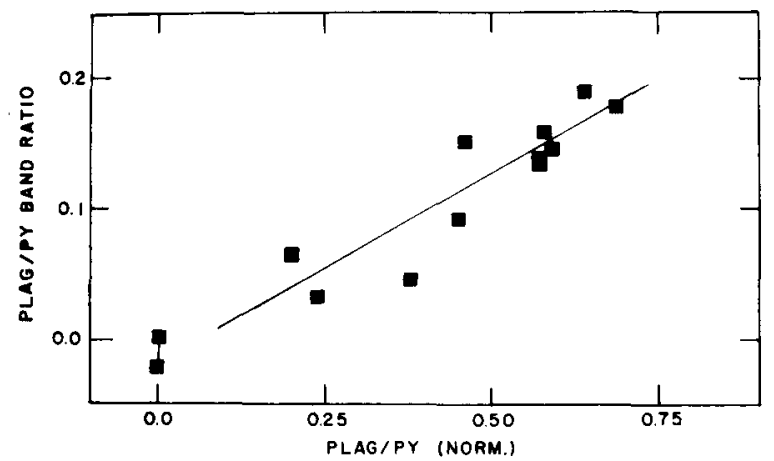

Figure 9. Correlation of plagioclase/pyroxene absorption band depth with plagioclase/pyroxene mineral abundance.

preted as diagnostic of a pigionite (pyroxene with moderate calcium content). The spectrum was matched to that of a Eucritic basaltic achondrite (Pyroxene + Plagioclase). A second pyroxene band was predicted near $2.0 \mu \mathrm{m}$.

b) Chapman and Salisbury (1973) compared this spectrum to a range of meteorites and concluded that it was best matched by the lab spectrum of Howarditic basaltic achondrite.

c) Veeder, Johnson and Matson (1975) measured a high resolution ( $\sim 50 \AA)$ 0.6 - $1.1 \mu \mathrm{m}$ reflectance spectrum of Vesta, determined the absorption band position to be $0.92 \pm 0.02 \mu \mathrm{m}$ and interpreted this to represent a calcic pyroxene or eucritic basaltic achondrite.

d) Johnson, Matson, Veeder and Loer (1975) measured the broad bandpass reflectance of Vesta at 1.65 and $2.20 \mu \mathrm{m}$ ( $\mathrm{H}$ and $\mathrm{K}$ filters) and concluded that that these data matched that expected for a basaltic achondritic surface material. They emphasized the need for higher resolution spectra beyond $1.0 \mathrm{\mu m}$.

e) Larson and Fink (1975) determined the $1.0-3.0 \mu \mathrm{m}$ reflectance of Vesta relative to the moon. (They invalidly assumed that the moon has a flat spectral reflectance curve at IR wavelengths - see for example Adams and McCord (1970) and Fink, Dekkers and Larson (1973, pp. L157). The effect is to produce too flat a spectral curve for Vesta and to assign a wavelength for the second pyroxene band that is somewhat high. (This problem has been corrected for more recent observations (Larson 1976, personal communication)).

They identified the predicted second pyroxene band and confirmed the existence of pyroxene in the surface material. They indicated that they did not see absorption bands for olivine, feldspar or ices. Using their position for the second band, they located a position of Adams (1974) band-band plot corresponding to a eucritic pigeonite $W_{15} \mathrm{Fs}_{75} \mathrm{En}_{10}$.

f) McFadden, McCord and Pieters (1976) have measured the high resolution $(20-40 \AA) 0.5-1.06 \mu \mathrm{m}$ spectrum and determined the band position to be $0.924 \pm$ $0.004 \mu \mathrm{m}$. (10-12 mole : Ca pyroxene). They suggest that the symmetry of the absorption feature indicates little or no olivine.

The improvementsin the mineralogical and petrological characterization of the surface material of 4 Vesta is the result of observational spectra with improved spectral resolution and coverage coupled with an improved understanding of the mineralogical significance of the spectral behavior of the reflectance curve. The recent effort has concentrated on characterizing the mineral absorption features more precisely. In the case of Vesta, this process is far advanced because of the relative ease of obtaining the observational data and because of the large amount of effort focused on this object. An equivalent effort on other asteroids would result in much improved definition of their surface materials as well. 


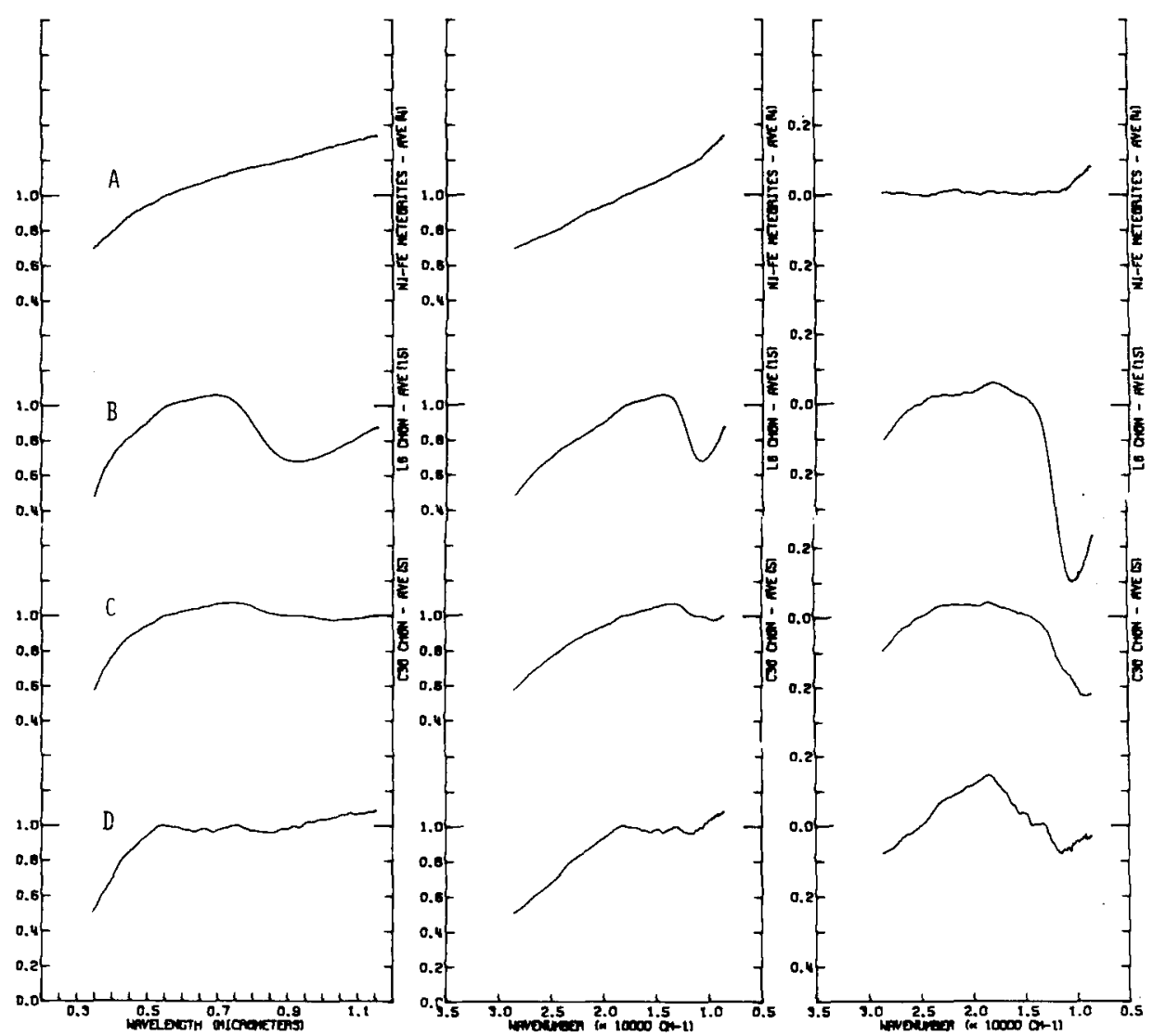

\footnotetext{
Figure 10 Normalized spectral reflectance curves of material whose spectral properties are indicative of general mineral classes. Each sample is plotted in three modes: normalized reflectance versus wavelength. versus wavenumber (energy) and the deviations from a linear continum fitted at 0.43 and $0.73 \mu m$ versus wavenumber. (a) Metallic $\mathrm{Ni}-\mathrm{Fe}-$ average of 4 iron meteorite spectra (note linear behavior in energy space above $7000 \mathrm{~cm}^{-1}$ ), (b) mafic silicate (olivine - pyioxene) assemblage - average of 15 L6 chondrite spectra (note strong curvatuie of continuum and strong UV falloff), (C) mafic silicate (olivine) plus opaque (carbon and/or magnetite) assemblage - average of 5 ( 30 (CO) carbonaceous chondrites (note curvature of continuum but weak IR features), and (d) clay mineral silicate + opaque (carbon

4 C2 (CM) carbonaceous chondrites (note position of break-in-slope

$(\sim 0.5 \mathrm{~mm})$ between flat $I R$ and steep blue-UV compared to $C 3$ spectrum).
}

\section{INTERPRETATION OF ASTEROIDAL SPECTRA}

McCord and Gaffey (1974) made the first systematic attempt to utilize diagnostic spectral features to interpret the spectra of a number of asteroids for mineral information. The spectra of 14 asteroids were interpreted and mineral assemblages similar to carbonaceous chondrites, basaltic achondrites, stonyirons and iron meteorites, were identified.

A very favorable apparition in early 1975 permitted the measurement of a 
variety of spectral data sets for the earth-approaching asteroid 433 Eros. Pieters, Gaffey, Chapman and McCord (1976) measured the 0.33 - $1.07 \mu \mathrm{m}$ spectral reflectance curve of Eros thru 25 narrow bandpass filters. This curve was interpreted to indicate an assemblage of olivine, pyroxene and metal, with metal abundance equal to or greater than that in the H-type chondrites. Veeder, Matson, Bergstralh and Johnson (1976) obtained the spectrum of Eros thru 11 filters from $0.65-2.2 \mu \mathrm{m}$ and concluded that their spectral data indicated a mixture of olivine and pyroxene with a metal-like phase. Wisniewski (1976) measured the spectrum of Eros $(0.4-1.0 \mu \mathrm{m}$ at higher resolution and concluded that this spectrum was best matched by a mixture of iron or stony-iron material with ordinary chondritic material (e.g.. iron + pyroxene + olivine) but suggested that olivine is absent or rare. Larson, Fink, Treffers and Gautier (1976) measured the $0.9-2.7 \mu \mathrm{m}$ high resolution spectral reflectance curve for Eros and identified $\mathrm{Ni}-\mathrm{Fe}$ and pyroxene but found no evidence of olivine and feldspar. The dispute over the olivine content arises because of slight differences in the observational spectra near $1 \mu \mathrm{m}$ and the uncertainty in the metal abundance is present because of incomplete quantitative understanding of the spectral contribution of metal in a mixture with silicates.

Gaffey and McCord (1976) have interpreted the spectral reflectance curves for about 65 asteroids. These spectra were classified into groups on the basis of mineralogically significant spectral parameters, such as the position, symmetry and intensity of absorption features, spectral continuum slope and curvature, spectral contrast and albedo. These groups were interpreted as follows:

a) Approximately $40 \%$ of these spectra (spectral types TA, TB, TC) exhibit a nearly flat curve longwards of $0.6 \mu \mathrm{m}$ with a relatively strong blue and UVfalloff shortwards of $0.5 \mu \mathrm{m}$ and a variable weak feature near $0.65 \mu \mathrm{m}$. Where available these asteroids are characterized by very low albedos (3-6\%). (A typical spectral curve is. shown on Figure lla) These spectral properties indicate an opaque-rich charge transfer $\left(\mathrm{Fe}^{2+}-\mathrm{Fe}^{3+}\right)$ silicate assemblage similar to the low temperature 'carbon plus clay mineral' assemblages of the $\mathrm{C} 1$ and $\mathrm{C} 2$ chondrites. The preservation of such an assemblage places an upper limit on any post accretionary thermal event of approximately $5000 \mathrm{C}$.

Several subdivisions of this group are distinguished on the basis of spectral behavior (increasing spectral contrast) in the IR. A similar variability is exhibited among the various laboratory reflectance spectra of the low metamorphic grade C-type meteorites, but the laboratory work to understand the reason for this variation is not complete. It is likely that it represents subtle variations in the composition of these materials. Several of these asteroids including 10 Hygiea (Zimmerman 1974, personal communication), 130 Elektra (Williams 1973) and 511 Davida (Zimmerman and Wetherill 1973) have been suggested as source bodies for Asteroid Belt material arriving at the Earth's surface, but an evaluation of this suggestion must await a detailed mineralogical and petrological comparison between the individual $\mathrm{C} 1$ and $\mathrm{C} 2$ meteorites and these asteroid surface materials.

b) Approximately $15 \%$ of these interpreted asteroids (spectral types TD, TE) have spectral reflectance curves characterized by significant UV-visible spectral contrast but lack significant IR spectral contrast or absorption features (Figure 11b), and have intermediate albedos $(9-15 \%)$. These spectral characteristics indicate a high temperature mafic silicate assemblage (olivine and/or pyroxene) with a relatively abundant opaque phase (magnetite and/or carbon) similar to $\mathrm{C} 3$ carbonaceous chondritic materials.

c) Approximately $10 \%$ of the interpreted asteroids (spectral type F) have spectra characterized by very low spectral contrast (flat) and a very short wavelength $(\sim 0.4 \mu \mathrm{m})$ edge of the UV falloff (Figure 1lc). The relatively high albedos ( 1 Ceres - 7\%, 2 Pallas - 8\% - Chapman, Morrison and Zellner 1975) and the spectral characteristics would rule out the ultra-primitive (e.g., very carbon rich) assemblages (Johnson and Fanale 1973) and would indicate instead a high 


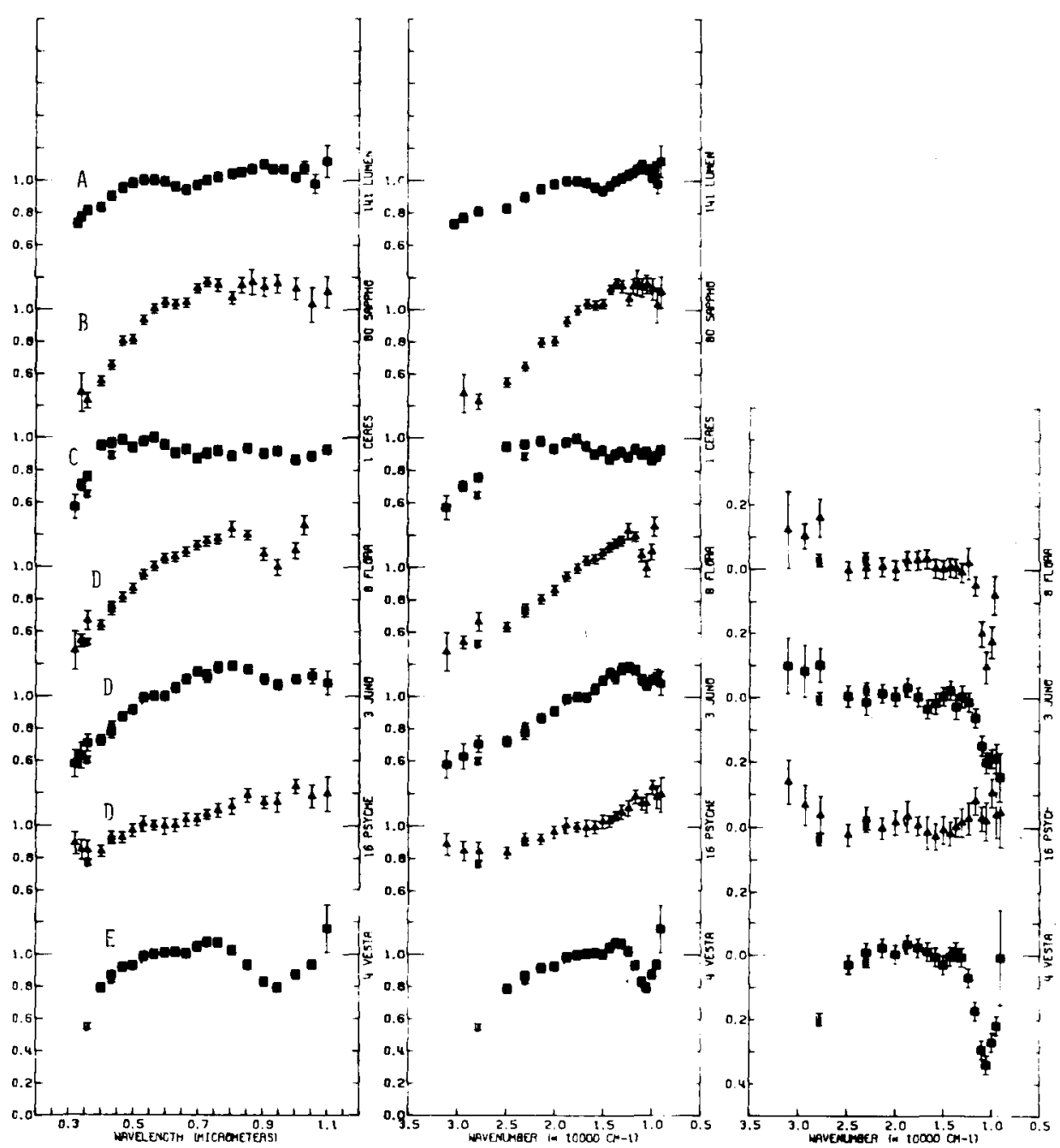

Figure 11 Normalized spectral reflectance curves for spectral type asteroids in same format as Figure 10. (a) 141 Lumen (TA, TB, TC), (b) 80 Sappho (TD, TE), (C) 1 Ceres (F) (d) 3 Juno, 8 Flora, 16 Psyche (R) (e) 4 Vesta.

temperature silicate assemblage (olivine and/or pyroxene) with excess opaque phases (magnetite + olivine such as the C4 meteorite 'Karoonda'). Such an assemblage would develop by post-accretionary heating and oxidation.

d) The majority of the rest of the interpreted spectra $(-30 \%)$ (spectra type $R$ ) exhibit the relatively linear blue and visible continuum indicating a significant spectral contribution by a $\mathrm{Ni}-\mathrm{Fe}$ phase (Figure 1ld). A variety of absorption feature shapes, locations and intensities near $1 \mu \mathrm{m}$ indicate a range of silicate compositions (olivine, pyroxene, olivine + pyroxene). The range of slopes of the continuum (shallow to steep) seems to indicate a range of metal particle sizes (fine $<1 \mathrm{~mm}$ to coarse $<5 \mathrm{~mm}$ ) in the substrate. These 
assemblages seem to represent a range of metal-rich (25-75\%) assemblages with silicates (e.g., stony-irons). A few of these type ' $R$ ' spectra, seem to represent either iron surfaces or iron + enstaiite ( E type chondrite) surfaces.

\section{ASTEROID SURFACE MATERIALS - IMPLICATIONS FOR THE ORIGIN OF METEORITES}

McCord, Adims and Johnson (1970) provided the first physical evidence that a link could exist between a specific meteorite class and a specific asteroid, when they showed that at least one asteroid (4 Vesta) was made of meteorite-like materials. Chapman and Salisbury (1973) and Johnson and Fanale (1973) did not find any significant number of matches between asteroidal spectra and meteorite spectra and concluded that either the majority of meteorites arriving at the earth's surface were derived from asteroids which had not been spectrally measured or some type of alternation process (space weathering or terrestrial alteration) was modifying the asteroid surface material or the meteorite samples such that the match could not be made. The problem was, complicated by the clear evidence that a strong bias was operating to select the meteorites arriving at the surface of the Earth, that is, ordinary chondrites constitute the major portion of the meteorites observed to fall (85.5\% - Mason 1962, p. 70) while observed belt asteroids with ordinary chondrite-like assemblages were rare or absent. The suggestion that two earth-crossing or earth-approaching asteroids (1685 Toro - Chapman, McCord and Pieters 1973, and 433 Eros - Pieters, Gaffey, Chapman and McCord 1976) resemble ordinary chondritic material would seem to indicate that the 'bias' is due to preferentially located source bodies for these meteorites.

Chapman, Morrison and Zellner (1975) noted that their type ' $\mathrm{C}$ ' asteroids became more relatively abundant from the inner edge of the Belt $(\sim 50 \%)$ to the outer Be1t $(\sim 90 \%)$. They assumed that ' $C$ ' was equivalent to 'carbonaceous chondrite' and proposed three models to produce this distribution: (a) a compositional variation in the condensing solar nebula with subsequent mixing of orbits, (b) inhomogeneous accretion with high temperature assemblage at the center being exposed in hajor collisional events or (c) differential breakup of melted and differentiated objects with strong metallic cores surviving.

Gaffey and McCord (1976) plot their mineralogical groups of asteroid surface materials as a function of semi-major axis (Figure 12) and conclude that there does exist evidence of a selection effect for high temperature assemblages in the inner Belt. The low temperature ' $\mathrm{C} 2$ ' assemblages are present across the Be1t. Williams (1976, personal communication) suggests that it is very unlikely that these low temperature assemblages were 'mixed' into their present distribution, but rather that the present orbits are quite similar to the original orbits. This produces an either/or situation vis-a-vis the $\mathrm{C} 1, \mathrm{C} 2, \mathrm{C} 3$ meteorites found in terrestrial collections. These meteorites show no evidence of any post-accretionary metamorphic event (Van Schmus 1969, Fuchs, 01sen and Jensen 1973, Kerridge and MacDougall 1976). Therefore, either

a) the asteroid surface materials which we interpret as 'C2 ' or 'C3' have never undergone any significant (e.g., a few hundred degrees Centigrade) thermal event in their post-accretionary history and coexist in a region where thermal events sufficient to melt and differentiate Vesta and the stony-iron asteroids occurred implying a very selective (on/off) heating mechanism, or

b) all of the asteroids have been exposed to some degree of post-accretionary heating (the present spectral data cannot determine this) but that none of the common ' $\mathrm{C} 2$ ' or ' $\mathrm{C} 3$ ' asteroidal material arrives at the earth's surface which would also seem to mitigate against the arrival of other (e.g., basaltic achondritic, stony-iron, etc.) materia! as well.

The former alternative seems the most reasonable. It does imply that the unmetamorphosed ' $\mathrm{C} 2$ ' and ' $\mathrm{C} 3$ ' materials derive from bodies formed by inhomogenous accretion, which have not been modified by either heating or by the out- 


\section{GAFFEY AND MCCORD}

RESONANCYSS WITH JUPITER

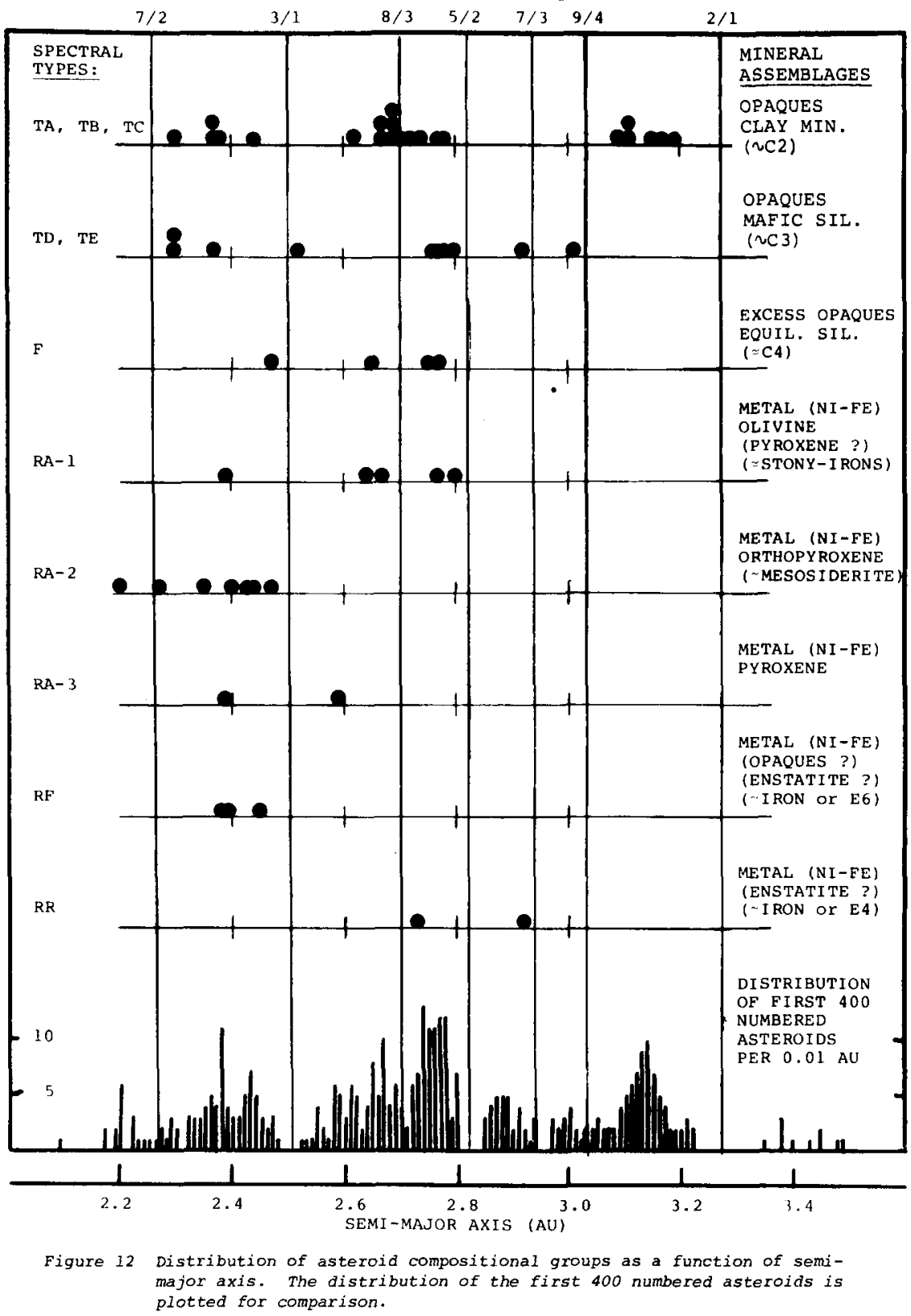

gassing of an interior being heated. Such outgassing $\left(\mathrm{H}_{2} \mathrm{~N}, \mathrm{CO}, \mathrm{CO}_{2}\right)$ during a heating event should modify the overlying strata and may be able to produce assemblages similar to $\mathrm{C} 4$ or Ureilite assemblages, while the differentiated 
meteorites develop in the interior. The selective heating episode could result from short-lived nuclides (e.g., A126) (Reeves and Adouze 1969) or solar wind heating (Sonett et al. 1970, Sonett 1971, Briggs 1976).

\section{CONCLUS IONS}

Based on the spectral evidence, it is plausible that most or all of the meteoritic material reaching the Earth's surface could be derived from asteroidal sources. Surface materials have been identified which have undergone the variety of accretionary and post-accretionary processes (or lack thereof) necessary to produce the known meteorite types, including direct accretion products, postaccretion alteration products and differentiated assemblages. The ' $\mathrm{Cl}-\mathrm{C} 2$ ' and ' $\mathrm{C} 3$ ' asteroid surface materials suggest that inhomogeneous accretion occurred for at least some objects.

The Main Belt asteroids could supply most types of known meteorites with the exception of the terrestrially-dominant ordinary chondrites, which are very rare or absent in the main belt. The ordinary chondrites appear to derive from earth-crossing or earth-approaching asteroids. If the ' $\mathrm{Cl}-\mathrm{C} 2$ ' assemblages present throughout the Belt represent accretion of primary condensate at that portion of the nebula and if the ordinary chondrites formed in a region of higher pressure and/or temperature than the $\mathrm{Cl}, \mathrm{C} 2$ and $\mathrm{C} 3$ materials, then it is probable that the parent bodies of the ordinary chondrites (>50 km, Wood 1967) accreted inside the orbit of the inner edge of the Main Belt $(<2.2 \mathrm{AU})$. The dynamic lifetime of a minor planet in an earth-crossing or approaching orbit is short compared to the age of the solar system $\left(\sim 10^{7}\right.$ years, wetheril1 1974$)$. If the relative abundance of ordinary chondritic material on such bodies is not merely a statistical fluke in the recent epoch, these short-1ived asteroids must be constantly replenished from a reservoir of parent bodies formed at less than $2.2 \mathrm{AU}^{\prime} \mathrm{s}$.

At the present time, no specific asteroid has been identified as the particular source body of a specific meteorite specimen present in a terrestrial collection. A detailed quantitative comparison of mineralogy, mineral chemistry and petrology between the meteorite specimens and the asteroid surface materials is necessary to evaluate any specific link. The asteroid surface materials cannot be characterized with sufficient precision with the present spectral data and interpretive sophistication to make such a comparison. Future effort should improve this situation.

\section{REFERENCES}

Adams, J. B. 1974, Jour. Geophys. Res., 79, 4829-4836.

Adams, J. B., and McCord, T. B. 1970, in Proc. Lunar Sci. Conf. 1st, 3, $1937-1945$. Be11, P. M., Mao, H. K., and Rossman, G. R. 1974, in Infrared and Raman Spectroscopy of Lunar and Terrestrial Minerals (ed. C. Karr) Academic Press, New York. Briggs, P. L. 1976, M.S. Diss., Mass. Inst. Tech., Cambridge, Mass.

Burns, R. G. 1970a, Mineralogical Applications of Crystal Field Theory, Cambridge University Press, New York.

Burns, R. G. 1970b, Amer. Mineral. 55, 1608-1632.

Chapman, C. R., Johnson, T. V., and McCord, T. B. 1971, in Physical Studies of Minor Planets (ed. T. Gehrels), NASA SP-267, 51-65.

Chapman, C. R., McCord, T. B., and Johnson, T. V. 1973, Astron. Jour. 78, 126140 .

Chapman, C. R., and Salisbury, J. W. 1973, Icarus, 19, 507-522.

Chapman, C. R., McCord, T. B., and Pieters, C. 1973, Astron. Jour. 78, 502-505.

Chapman, C. R., Morrison, D., and Ze1lner, B. 1975, Icarus, 25, 104-130.

Chapman, C. R., and Morrison, D. 1976, Icarus, 28, 91-94. 
Fink, U., Dekkers, N. H., and Larson, H. P. 1973, Astrophys. Jour. 79, L155-L159. Fuchs, L., O1sen, E., and Jensen, K. J. 1973, Smithsonian Contrib. Earth SCi. 10, Gaffey, M. J. 1974, Ph.D. Diss., Mass. Inst. Tech., Cambridge, Mass.

Gaffey, M. J. 1976, Jour. Geophys. Res., 81, 905-920.

Gaffey, M. J., and McCord, T. B. 1976, in preparation.

Gehrels, T. 1970, in Surfaces and Interiors of planets and Satellites, (ed. A. Dollfus), pp. 317-375, Academic Press, Inc., London.

Hapke, B. 1971, in Physical Studies of Minor Planets, (ed. T. Gehrels), NASA SP-267, p. $67-77$.

Johnson, T. V., and Fanale, F. P. 1973, Jour. Geophys. Res., 78, 8507-8518.

Johnson, T. V., Matson, D. L., Veeder, G. J., and Loer, S. J. 1975, Astrophys. Jour., 197, 527-531.

Kerridge, J. F., and MacDouga11, J. D. 1976, Earth Planet. Sci. Lett., 29, 341348 .

Larson, H. P. 1976 (abstract), I.A.U. Col1. No. 39, Lyon, France.

Larson, H. P., and Fink, U. 1975, Icarus, 26, 420-427.

Larson, H. P., Fink, U., Treffers, R. R., and Gautier, T. N. 1976, Icarus, 28, 95-103.

Mason, B. 1962, Meteoritics, John Wiley, New York.

Mason, B. 1971, Meteoritics, 6, 59-70.

Matson, D. L., Johnson, T. V., and Veeder, G. J. 1976, (abstract), T.A.U. Co11. No. 39, Lyon, France.

McCord, T. B., Adams, J. B., and Johnson, T. V. 1970, Science, 168, 1445-1447.

McCord, T. B., and Gaffey, M. J. 1974, Science, 186, 352-355.

McCord, T. B., and Chapman, C. R. 1975a, Astrophys. Jour. 195, 553-562.

McCord, T. B, , and Chapman, C. R. 1975b, Astrophys. Jour. 197, 781-790.

McFadden, L., McCord, T. B., and Pieters, C. 1976, submitted to Icarus.

Nash, D. B., and Cone1, J. F. 1974, Jour. Geophys. Res., 79, 1615-1621.

Pieters, C., Gaffey, M. J., Chapman, C. R., and McCord, T. B. 1976, Icarus, 28, $105-115$.

Reeves, H., and Audouze, J. 1969, Earth Planet. Sci. Lett., 4, 135.

Sal isbury, J. W., and Hunt, G. R. 1974, Jour. Geophys. Res., 79, 4439-4441.

Sonett, C. P. 1971, in Physical studies of Minor Planets (ed. T. Gehrels), NASA SP-267, pp. 239-245.

Sonett, C. P., Colburn, D. S., Schwartz, K., and Keil, K. 1970, Astrophys. Space Sci., 7, 446.

Van Schmus, W. R. 1969, in Meteorite Research. (ed. P. Mil1man), pp. 480-491, D. Reidel Publishing Company, Dordrecht, Holland.

Veeder, G. J., Matson, D. L., Bergstralh, J. T., and Johnson, T. V. 1976, Icarus, 28, 79-85.

Wetheril1, G. W. 1974, in Ann. Rev. Earth Planet. Sci., (ed. F. A. Donath), Vol. 2, 303-331.

White, W. B., and Keester, K. L. 1966, Amer. Mineral., 51, 774-791.

White, W. B., and Keester, K. L. 1967, Amer. Mineral., 52, 1508-1514.

Williams, J. G. 1973, (abstract), EOS Trans. Amer. Geophys. Union, 54, 233.

Wisniewski, W. Z. 1976, Icarus, 28, 87-90.

Wood, J. A. 1967, Icarus, 6, 1-49.

Ze11ner, B. 1975, Astrophys. Jour. Lett., 198, L45-L47.

Zeliner, B., Wisniewski, W. Z., Andersson, L., and Bowel1, E. 1975, Astron. Jour. 80, 986-995.

Zimmerman, P. D., and Wetheri11, G. W. 1973, Science, 182, 51-53.

\section{DTSCUSSION}

EL GORESY: How far does the variation in the oxidation state of $3 d$ transition elements influence the accuracy of identification of pyroxenes? 
GAFFEY: The variation of oxidation states for the transition metals is quite sensitively indicated in the reflectance spectra, introducing additional features generally near $0.5-0.7 \mu \mathrm{m}$. Variations in these oxidation states can be diagnosed from the spectra as shown for a nucleus of pyroxenes by Adams (1975, in IR and Raman Spectroscopy of Lunar and Terrestrial Minerals).

since any cation which modifies the crystal structure (increases size of crystal lattice sites) or distribution of transition metal cations has a spectral effect, there is no major problem with redundancy (e.g., the same spectral reflectance curve from two distinctly different chemistries).

EL GORESY: How do you determine the crystal field spectra of opaques e.g., magnetite?

GAFFEY: One can measure the lab spectra for ultrafine opaques (e.g., $500 \AA$ particles for magnetite) or for fine grained opaques dispersed in a neutral carrier. One can also use Molecular Orbital calculations (e.g., Burns, Loefflen and co-workers) for known crystal lattice structures and cation distributions to calculate the energies of electronic transitions. (Keep in mind, that the term "opaque" simply means that at the appropriate particle size the dimensions represent many optical depths at the interesting wavelengths.

GROSSMAN: What causes the spectral differences between $C 1$ and $C 2$ chondrites?

GAFFEY: The detailed laboratory and theoretical investigation of the relationship of spectral properties to mineralogy and petrology for the entire group of dark meteoritic mineral assemblages (e.g., Cl, C2, C30, C3U, C4, Ureilite, black chondrites, E4 chondrite) is in an early stage, so that we cannot give a specific answer to that question. We also have a problem in that good spectral reflectance data is available for only one $C l$ meteorite (Orgueil) so we are not certain how typical this spectrum is of the $C l$ chondrites.

GROSSMAN: What do you mean by disequilibrium silicates?

GAFFEY: We use the term "disequilibrium silicates" to mean the unequilibrated (relative to the higher metamorphic grade meteorites) layer lattice silicates on clay minerals, of the $C 1$ and $C 2$ meteoritic mineral assemblages which contain roughly equal (with a factor of 3 or 4) amounts of bivalent and trivalent iron $\left(\mathrm{Fe}^{2+} \& \mathrm{Fe}^{3+}\right)$ in the silicate structure.

ANDERS: You have seen some asteroids resembling mesosiderites (metal and pyroxene) but apparently more resembling pallasites (metal and olivine), although the latter are at least twice as abundant among meteorite finds. Is this a matter of observational selection, due to the spectroscopic inconspicuousness of olivine?

GAFFEY: In our discussion, we concentrated on the basis of the interpretation technigue and the asteroid types discussed were mentioned as specific examples. Among the $\sim 65$ good spectra which we have examined, a significant number (-5) exhibit the spectral characteristics of a dominantly metal-plus-olivine assemblage.

WETHERILL: On the basis of some quantitative calculations $I$ have made, it seems to me that the coverage of asteroid spectra is becoming sufficiently complete that the paucity of bodies with spectra resembling the ordinary chondrites is likely to be significant. It does not help that some dynamically short-lived Mars and Earth crossers resemble chondrites, as these require a source of similar composition. The astercid which many workers have identified with high grade hypersthene chondrite, 349 Dembowska has a prominent $.65 \mu m$ band which is 


\section{GAFFEY AND MCCORD}

not seen in these chondrites, and this may be an indication that even this object may differ from chondrites.

CHAPMAN: The latest work on the stellar calibrations of asteroid spectrophotometry suggests that the previously published asteroid spectra show spuriously exaggerated absorptions near $0.65 \mu \mathrm{m}$. I think that the only objects that we can be sure exhibit $0.65 \mu \mathrm{m}$ bands are those asteroids with spectra analogous to those of $\mathrm{C2}$ meteorites. 